$\breve{P}$ Note 497

Debuncher Momentum Aperture

Measurements

S. O'Day 


\begin{abstract}
During the November 1990 through January $1991 \bar{p}$ studies period, the momentum aperture of the beam in the debuncher ring was measured. The momentum aperture $(\Delta p / p)$ was found to be $4.7 \%$. The momentum spread was also measured with beam bunch rotation off. A nearly constant particle population density was observed for particles with $\Delta p / p$ of less than $4.3 \%$, indicating virtually unobstructed orbits in this region. The population of particles with momenta outside this aperture was found to decrease rapidly. An absolute or "cut-off" momentum aperture of $\Delta p / p=5.50 \%$ was measured.

\section{Momentum Aperture}

After "reverse" direction proton beam was injected into the debuncher from the accumulator, the beam was bunched with the DRF3 rf. After the capture, the frequency was increased until a small increase in frequency $(.002 M H z)$ resulted in a large $(30 \%)$ beam loss. The frequency was then decreased until the same thing happened at a lower frequency. The beam current versus if frequency is plotted in figure 1. The aperture limits are marked in the figure. Since the frequency aperture is related to the momentum aperture by

$$
\frac{\Delta p}{p}=\frac{1}{\eta} \frac{\Delta f}{f} \text { where } \eta=\text { dispersion }=.0056
$$
\end{abstract}

the momentum aperture is $4.7 \%$ from the limits shown in figure 1 .

\title{
Momentum Spread
}

Using anti-protons with the bunched beam rotation off, the natural momentum spread of the debuncher beam can be measured using a frequency spectrum analyzer. In figure 2, a plot of the logarithm of the current versus the beam frequency is shown. By measuring the narrowest frequency width(see figure), one 
may obtain the momentum aperture inside which particles move without encountering obstruction. Outside this aperture, there is a rapidly declining particle population until an absolute aperture limit is hit. Using once again $\frac{\Delta p}{p}=\frac{1}{\eta} \frac{\Delta f}{f}$ gives us

$$
\begin{aligned}
\frac{\Delta p}{p}_{\text {un-obstructed }} & =4.3 \% \text { and } \\
\frac{\Delta p}{p_{\text {absolute }}} & =5.5 \%
\end{aligned}
$$

In summary, a description of the methods by which the momentum aperture and natural momentum spread were measured has been presented and the values measured during the $1990-1991 \bar{p}$ studies have been given, 


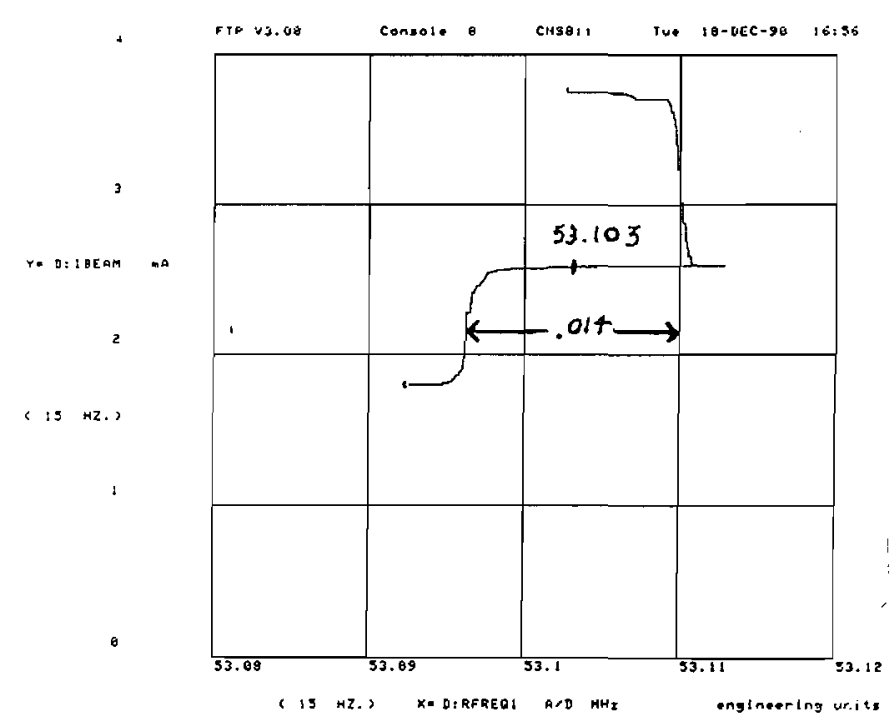

Figure 1. Debuncher beam momentum aperture limits.

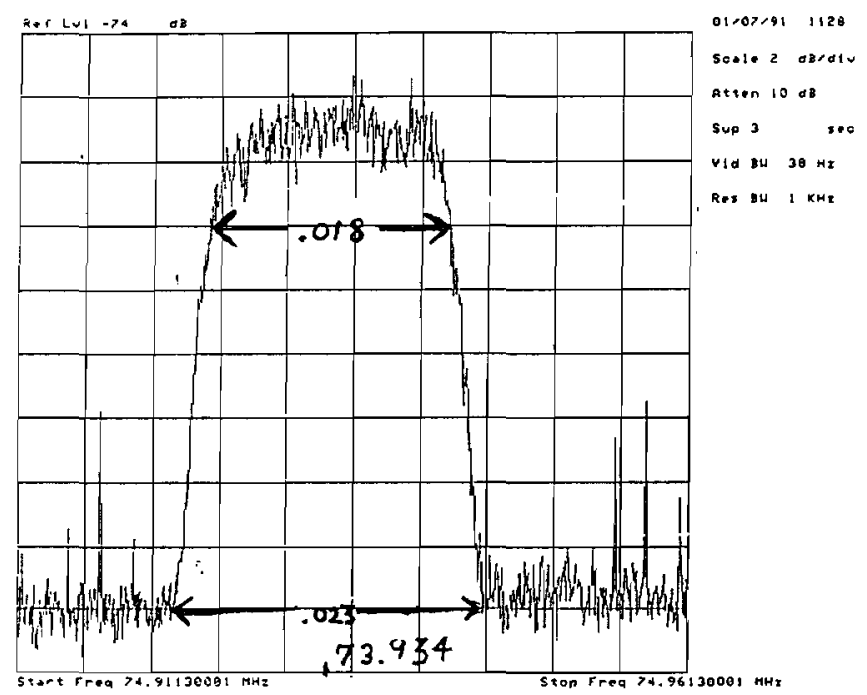

Figure 2. Debuncher beam momentum spread. 